\title{
GNSS Ambiguity Resolution: When and How to Fix or not to Fix?
}

\author{
P.J.G. Teunissen, S. Verhagen (invited paper) \\ Delft Institute of Earth Observation and Space systems (DEOS), Delft University of Technology, \\ Kluyverweg 1, 2629 HS Delft, The Netherlands, e-mail: P.J.G. Teunissen@TUDelft.nl
}

\begin{abstract}
In order to facilitate rapid and precise GNSS positioning, the integer carrier phase ambiguities need to be resolved. Since wrong integer ambiguity estimates may result in fixed position estimates which are worse than their float counterparts, very high success rates (i.e. high probabilities of correct integer estimation) or very low failure rates are required when performing ambiguity resolution. We discuss two different approaches of ambiguity resolution, a model-driven approach and a data-driven approach. The first is linked to the theory of integer estimation and the second is linked to the theory of integer aperture estimation. In the first approach, the user chooses an integer estimator and computes on the basis of his/her model the corresponding failure rate. The decision whether or not to use the integer ambiguity solution is then based on the thus computed value of the failure rate. This approach is termed model-driven, since the decision is solely based on the strength of the underlying model and not dependent on the actual ambiguity float estimate. This approach is simple and provides a priori information on the outcome of the decision process. A disadvantage of the model-driven approach is that it does not provide the user of any control over the failure rate. This disadvantage is absent when one uses the more elaborate data-driven approach of integer aperture estimation. With this approach the user sets his/her own failure rate (irrespective of the strength of the underlying model), thus generating an aperture space which forms the basis of the decision process: the integer solution is chosen as output if the float solution resides inside the aperture space, otherwise the float solution is maintained. Although more elaborate, the data-driven approach is more flexible than the model-driven approach and can provide a guaranteed failure rate as set by the user.

In this contribution we compare the model-driven and data-driven approaches, describe the decision making process of when and how to fix (or not to fix) and also give the optimal data-driven approach. We
\end{abstract}

also show how the so-called 'discrimination tests', in particular the popular 'ratio test', fit into this framework. We point out that the common rationales for using these 'tests' are often incorrectly motivated in the literature and we show how they should be modified in order to reach an overall guaranteed failure rate for ambiguity resolution.

Keywords. Integer least-squares, integer aperture estimation

\section{GNSS Ambiguity Resolution}

\subsection{The GNSS Model}

As our point of departure we will take the following system of linear(ized) observation equations

$$
y=A a+B b+e
$$

where $y$ is the given GNSS data vector of order $m$, $a$ and $b$ are the unknown parameter vectors respectively of order $n$ and $p$, and where $e$ is the noise vector. In principle all the GNSS models can be cast in this frame of observation equations. The data vector $y$ will usually consist of the 'observed minus computed' double-difference (DD) phase and/or pseudorange (code) observations accumulated over all observation epochs. The entries of vector $a$ are then the DD carrier phase ambiguities, expressed in units of cycles rather than range. They are known to be integers, $a \in Z^{n}$. The entries of the vector $b$ will consist of the remaining unknown parameters, such as for instance baseline components (coordinates) and possibly atmospheric delay parameters (troposphere, ionosphere). They are known to be real-valued, $b \in R^{p}$.

The procedure which is usually followed for solving the GNSS model (1), can be divided into three steps. In the first step one simply disregards the integer constraints $a \in Z^{n}$ on the ambiguities and 
applies a standard least-squares adjustment, resulting in real-valued estimates of $a$ and $b$, together with their variance-covariance (vc-) matrix

$$
\left[\begin{array}{c}
\hat{a} \\
\hat{b}
\end{array}\right], \quad\left[\begin{array}{cc}
Q_{\hat{a}} & Q_{\hat{a} \hat{b}} \\
Q_{\hat{b} \hat{a}} & Q_{\hat{b}}
\end{array}\right]
$$

This solution is referred to as the 'float' solution. In the second step the 'float' ambiguity estimate $\hat{a}$ is used to compute the corresponding integer ambiguity estimate $\check{a}$. This implies that a mapping $S: R^{n} \mapsto$ $Z^{n}$, from the $n$-dimensional space of reals to the $n$ dimensional space of integers, is introduced such that

$$
\check{a}=S(\hat{a})
$$

Once the integer ambiguities are computed, they are used in the third step to finally correct the 'float' estimate of $b$. As a result one obtains the 'fixed' solution

$$
\check{b}=\hat{b}-Q_{\hat{b} \hat{a}} Q_{\hat{a}}^{-1}(\hat{a}-\check{a})
$$

This three-step procedure is still ambiguous in the sense that it depends on which mapping $S$ is chosen.

\subsection{The Class of Integer Estimators}

If one requires the output of the map $S$ to be integer, $S: R^{n} \mapsto Z^{n}$, then $S$ will not be one-to-one due to the discrete nature of $Z^{n}$. Instead it will be a manyto-one map. This implies that different real-valued vectors will be mapped to one and the same integer vector. One can therefore assign a subset $S_{z} \subset R^{n}$ to each integer vector $z \in Z^{n}$ :

$$
S_{z}=\left\{x \in R^{n} \mid z=S(x)\right\}, \quad z \in Z^{n}
$$

The subset $S_{z}$ contains all real-valued vectors that will be mapped by $S$ to the same integer vector $z \in$ $Z^{n}$. This subset is referred to as the pull-in region of $z$. It is the region in which all vectors are pulled to the same integer vector $z$.

Since the pull-in regions define the integer estimator completely, one can define classes of integer estimators by imposing various conditions on the pull-in regions. One such class was introduced in (Teunissen, 1999a) as follows.

Definition 1 (Integer estimators). The mapping $\check{a}=S(\hat{a})$, with $S: R^{n} \mapsto Z^{n}$, is said to be an integer estimator if its pull-in regions satisfy

$$
\begin{cases}\cup_{z \in Z^{n}} S_{z}=R^{n} & \\ \operatorname{Int}\left(S_{u}\right) \cap \operatorname{Int}\left(S_{z}\right)=\emptyset, & \forall u, z \in Z^{n}, u \neq z \\ S_{z}=z+S_{0}, & \forall_{z} \in Z^{n}\end{cases}
$$

This definition is motivated as follows. The first condition states that the pull-in regions should not leave any gaps and the second that they should not overlap. The third condition of the definition follows from the requirement that $S(x+z)=S(x)+z, \forall x \in R^{n}$, $z \in Z^{n}$.

Using the pull-in regions, one can give an explicit expression for the corresponding integer estimator $\breve{a}$. It reads

$\check{a}=\sum_{z \in Z^{n}} z s_{z}(\hat{a})$ with $s_{z}(\hat{a})= \begin{cases}1 & \text { if } \hat{a} \in S_{z} \\ 0 & \text { if } \hat{a} \notin S_{z}\end{cases}$

The three best known integer estimators are integer rounding, integer bootstrapping and integer leastsquares. The latter is shown to be optimal, cf. (Teunissen, 1999a), which means that the probability of correct integer estimation is maximized.

The integer least-squares (ILS) estimator is defined as

$$
\check{a}_{\mathrm{LS}}=\arg \min _{z \in Z^{n}}\|\hat{a}-z\|_{Q_{\hat{a}}}^{2}
$$

with the squared norm $\|\cdot\|_{Q}^{2}=(\cdot)^{T} Q^{-1}(\cdot)$.

In contrast to integer rounding and integer bootstrapping, an integer search is needed to compute $\check{a}_{\mathrm{LS}}$. The ILS procedure is mechanized in the LAMBDA method, which is currently one of the most applied methods for GNSS carrier phase ambiguity resolution, see e.g. (Teunissen, 1993; Hofmann-Wellenhoff and Lichtenegger, 2001; Strang and Borre, 1997; Teunissen, 1998; Misra and Enge, 2001).

As mentioned above, the ILS estimator maximizes the probability of correct integer estimation, referred to as the success rate, which is given by:

$$
P_{S, \mathrm{ILS}}=P(\check{a}=a)=\int_{S_{a}} f_{\hat{a}}(x) \mathrm{d} x
$$

where $P(\check{a}=a)$ is the probability that $\check{a}=a$, and $f_{\hat{a}}(x)$ is the probability density function of the float ambiguities, for which in practice the normal (Gaussian) distribution is used. Hence, the success rate can be evaluated without the need for actual data, since it can be computed once the vc-matrix $Q_{\hat{a}}$ is known.

\section{Model-Driven Approach}

The precision of the fixed baseline solution can be shown to be much better than the precision of its float counterpart, provided that the success rate is close to 1 and thus the failure rate, $P_{f}=1-P_{s}$, must be close 
to 0 . Hence, it can be argued that the fixed solution should only be used if this is known to be the case.

The model-driven approach to integer estimation is a three-step procedure:

1. Apply an integer map such that $\check{a}=S(\hat{a})$

2. Evaluate the failure rate $P_{f}=P(\check{a} \neq a)=1-$ $P(\check{a}=a)$

3. Decision:

$$
\text { If } \begin{cases}P_{f} \leq \epsilon & \text { use } \check{a} \\ P_{f}>\epsilon & \text { use } \hat{a}\end{cases}
$$

where $\epsilon$ is a user-defined threshold, e.g. $\epsilon=10^{-2}$.

Obviously, this model-driven approach is simple and valid, since the failure rate can be determined prior to the actual integer estimation step. Hence, also the decision whether or not to fix can be made a priori. The choice on how to fix is also clear: the integer least-squares estimator is optimal since it minimizes the failure rate. An additional advantage is that an overall and rigorous quality description of the fixed solution $(\check{a}$ and $\breve{b})$ is available, cf. (Teunissen, 1999b, 2002).

Disadvantages of the model-driven approach are that the sample $\hat{a}$ has no influence on the decision and that the user has no control over the failure rate except for strengthening the model. The first disadvantage implies that there might be one or more other integer candidates which is/are almost as likely to be the correct solution. Therefore in practice often a discrimination test is applied as an (additional) decision tool, see section 3.1.

Strengthening the model may only be an option in the design phase of a measurement campaign, but not after the data is collected.

\section{Data-Driven Approach}

\subsection{Discrimination Tests}

In literature several discrimination tests have been proposed in order to decide whether or not to fix the ambiguities. A review and evaluation of the tests can be found in Verhagen (2004). Well-known examples are the ratio test, distance test and projector test. Among the most popular tests is the ratio test, see e.g. (Euler and Schaffrin, 1991; Wei and Schwarz, 1995; Han and Rizos, 1996; Leick, 2003), where the decision is made as follows:

1. Apply ILS to obtain $\check{a}$ and $\check{a}_{2}$

2. Evaluate ratio $\frac{\|\hat{a}-\check{a}\| \|_{Q_{\hat{a}}}^{2}}{\left\|\hat{a}-\check{a}_{2}\right\|_{Q_{\hat{a}}}^{2}}$
3. Decision:

$$
\text { If } \frac{\|\hat{a}-\check{a}\|_{Q_{\hat{a}}}^{2}}{\left\|\hat{a}-\check{a}_{2}\right\|_{Q_{\hat{a}}}^{2}} \begin{cases}\leq \delta & \text { use } \check{a} \\ >\delta & \text { use } \hat{a}\end{cases}
$$

where $\check{a}_{2}$ is the second-best integer solution in the ILS sense. Obviously, the decision is data-driven. Note that in practice the reciprocal of the test statistic is mostly used.

It is, however, not clear what role is actually played by this ratio test. The common motivation for using it is that it is a validation test, i.e. that it tells the user whether or not the fixed solution is true or false. This is not correct. Moreover, the current ways of choosing the threshold value $\delta$ are ad hoc or based on false theoretical grounds, see (Teunissen and Verhagen, 2004). Often a fixed value of $\frac{1}{2}$ or $\frac{1}{3}$ is used.

A problem with the ratio test, or any other discrimination test, is that the model-driven failure rate is not applicable anymore, since the test implicitly introduces a probability of not fixing. Another implication is that the overall quality of the fixed solution cannot be evaluated when the ratio test is included, whereas with the model-driven approach this is possible.

\subsection{Integer Aperture Estimation}

In practice, a user will decide not to use the fixed solution if either the probability of failure is too high, or if the discrimination test is not passed. This gives rise to the thought that it might be interesting to use an ambiguity estimator defined such that three situations are distinguished: success if the ambiguity is fixed correctly, failure if the ambiguity is fixed in correctly, and undecided if the float solution is maintained. This can be accomplished by dropping the condition that there are no gaps between the pullin regions, so that the only conditions on the pull-in regions are that they should be disjunct and translational invariant. Then integer estimators can be determined that somehow regulate the probability of each of the three situations mentioned above.

The new class of ambiguity estimators was introduced in Teunissen (2003a, c), and is called the class of Integer Aperture (IA) estimators.

Definition 2. (Integer aperture estimators) The integer aperture estimator, $\bar{a}$, is defined as:

$$
\bar{a}=\sum_{z \in \mathbb{Z}^{n}} z \omega_{z}(\hat{a})+\hat{a}\left(1-\sum_{z \in \mathbb{Z}^{n}} \omega_{z}(\hat{a})\right)
$$


with the indicator function $\omega_{z}(x)$ defined as:

$$
\omega_{z}(x)= \begin{cases}1 & \text { if } x \in \Omega_{z} \\ 0 & \text { otherwise }\end{cases}
$$

The $\Omega_{z}$ are the aperture pull-in regions, which have to fulfill the following conditions (compare with Definition 1):

$$
\begin{cases}\bigcup_{z \in \mathbb{Z}^{n}} \Omega_{z}=\Omega & \\ \operatorname{Int}\left(\Omega_{u}\right) \bigcap \operatorname{Int}\left(\Omega_{z}\right)=\emptyset, & \forall u, z \in \mathbb{Z}^{n}, u \neq z \\ \Omega_{z}=z+\Omega_{0}, & \forall z \in \mathbb{Z}^{n}\end{cases}
$$

$\Omega \subset \mathbb{R}^{n}$ is called the aperture space. From the first condition follows that this space is built up of the $\Omega_{z}$. The second and third conditions state that these aperture pull-in regions must be disjunct and translational invariant.

Figure 1 shows a two-dimensional example of aperture pull-in regions that fulfill the conditions in Definition 2, together with the ILS pull-in regions.

So, when $\hat{a} \in \Omega$ the ambiguity will be fixed using one of the admissible integer estimators, otherwise the float solution is maintained. This means that indeed the following three cases can be distinguished:

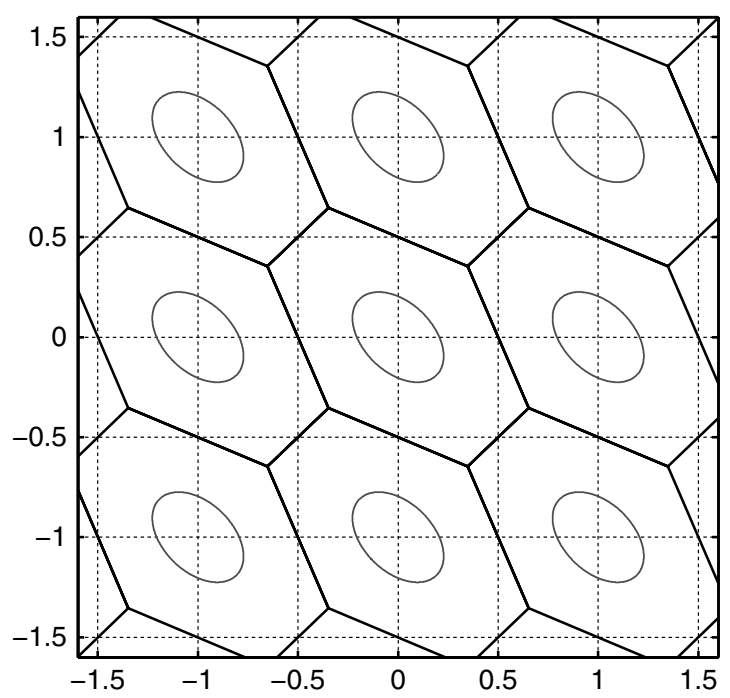

Fig. 1. Two-dimensional example of aperture pull-in regions (ellipses), together with the ILS pull-in regions (hexagons).
$\hat{a} \in \Omega_{a}$
success: correct integer estimation
$\hat{a} \in \Omega \backslash \Omega_{a}$
$\hat{a} \notin \Omega$
failure: incorrect integer estimation
undecided: ambiguity not fixed

The corresponding probabilities of success $(s)$, failure $(f)$ and undecidedness $(u)$ are given by:

$$
\begin{aligned}
& P_{s}=P(\bar{a}=a)=\int_{\Omega_{a}} f_{\hat{a}}(x) \mathrm{d} x \\
& P_{f}=\sum_{z \in \mathbb{Z}^{n} \backslash\{a\}_{\Omega_{z}}} \int_{\hat{a}}(x) \mathrm{d} x \\
& P_{u}=1-P_{s}-P_{f}=1-\int_{\Omega_{0}} f_{\check{\epsilon}}(x) \mathrm{d} x
\end{aligned}
$$

The first two probabilities are referred to as success rate and failure rate respectively. Note the difference with the ILS success rate given in equation (8), where the integration is over the ILS pull-in region $S_{a} \supset$ $\Omega_{a}$. The expression for the failure rate is obtained by using the probability density function of the ambiguity residuals $\check{\epsilon}=\hat{a}-\check{a}$ :

$$
f_{\breve{\epsilon}}(x)=\sum_{z \in \mathbb{Z}^{n}} f_{\hat{a}}(x+z) s_{0}(x)
$$

with $s_{0}(x)=1$ if $x \in S_{0}$ and $s_{0}(x)=0$ otherwise, cf. Teunissen (2002); Verhagen and Teunissen (2004).

\subsection{Fixed Failure Rate Approach}

As mentioned in the beginning of this section, for a user it is especially important that the probability of failure, the failure rate, is below a certain limit. The approach of integer aperture estimation allows us now to choose a threshold for the failure rate, and then determine the size of the aperture pull-in regions such that indeed the failure rate will be equal to or below this threshold. So, applying this approach means that implicitly the ambiguity estimate is validated using a sound criterion. However, there are still several options left with respect to the choice of the shape of the aperture pull-in regions.

It is very important to note that Integer Aperture estimation with a fixed failure rate is an overall approach of integer estimation and validation, and allows for an exact and overall probabilistic evaluation of the solution. With the traditional approaches, e.g. the Ratio Test applied with a fixed critical value, this is not possible. 


\subsection{ILS + Ratio Test is Integer Aperture Estimator}

Despite the criticism on the Ratio Test given in section 3.1 it is possible to give a firm theoretical basis for this test, since it can be shown that the procedure underlying the Ratio Test is a member from the class of integer aperture estimators. The acceptance region or aperture space is given as:

$$
\begin{gathered}
\Omega=\left\{x \in \mathbb{R}^{n} \mid\|x-\check{x}\|_{Q_{\hat{a}}}^{2} \leq \delta\left\|x-\check{x}_{2}\right\|_{Q_{\hat{a}}}^{2},\right. \\
0<\delta \leq 1\}
\end{gathered}
$$

with $\check{x}$ and $\check{x}_{2}$ the best and second-best ILS estimator of $x$. Let $\Omega_{z}=\Omega \bigcap S_{z}$, i.e. $\Omega_{z}$ is the intersection of $\Omega$ with the ILS pull-in region. Then all conditions of Defintion 2 are fulfilled, since:

$$
\begin{aligned}
\Omega_{0}= & \left\{x \in \mathbb{R}^{n} \mid\|x\|_{Q_{\hat{a}}}^{2} \leq \delta\|x-z\|_{Q_{\hat{a}}}^{2},\right. \\
& \left.\forall z \in \mathbb{Z}^{n} \backslash\{0\}\right\} \\
\Omega_{z}= & \Omega_{0}+z, \forall z \in \mathbb{Z}^{n} \\
\Omega= & \bigcup_{z \in \mathbb{Z}^{n}} \Omega_{z}
\end{aligned}
$$

The proof was given in Teunissen (2003b).

The acceptance region of the Ratio Test consists thus of an infinite number of regions, each one of which is an integer translated copy of $\Omega_{0} \subset S_{0}$. The acceptance region plays the role of the aperture space, and $\delta$ plays the role of aperture parameter since it controls the size of the aperture pull-in regions.

Compared to the approach in section 3.1 an important difference is that $\delta$ is now based on the modeldriven failure rate. Hence, before the decision step, an additional step is required to determine $\delta$ based on the user-defined choice $P_{f}=\beta$. This implies that a probabilistic evaluation of the solution can be made, see section 3.3.

As an illustration of the difference between the traditional and the Integer Aperture approach with the Ratio Test, five dual-frequency GPS models are considered. Based on Monte-Carlo simulations the success and failure rates as function of $\delta$ are determined for each of the models, see Figure 2. It can be seen that with a fixed value of $\delta=0.3$ (close to the value of $\frac{1}{3}$ often used in practice) for most of the models considered here a very low failure rate is obtained, but that this is not guaranteed. This seems good, but at the same time also the corresponding success rate is low. If the threshold value would have been based on a fixed failure rate of e.g. 0.005 , the corresponding
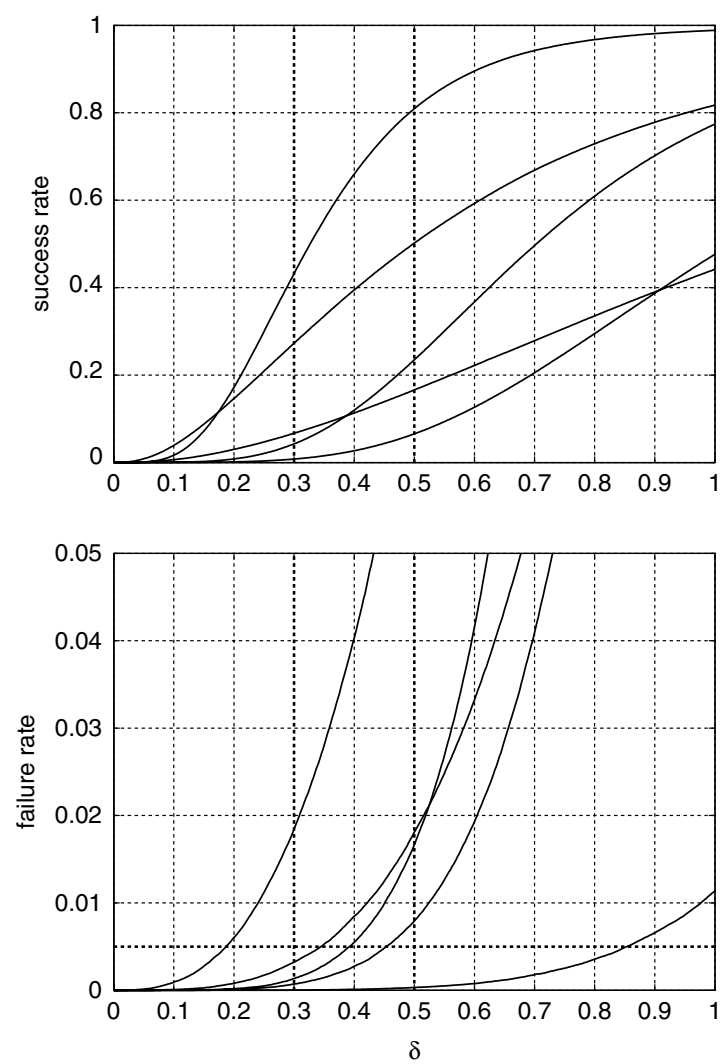

Fig. 2. Success and failure rates as function of the threshold value $\delta$ for 5 GPS models.

$\delta$ would have been very different for each of the models, and in most cases larger than 0.3 , and thus a higher success rate and higher probability of a fix $\left(=P_{s}+P_{f}\right)$ would be obtained. Hence, the integer aperture approach with fixed failure rate is to be preferred.

\subsection{Optimal Integer Aperture Estimation}

The approach of integer aperture estimation with a fixed failure rate has two important advantages. The first is that IA estimation can always be applied, independent of the precision, since the user does not have to be afraid that the failure rate is too high. The second advantage is that for the first time sound theoretical criteria are available for the validation of the estimates. For that purpose, the Ratio Test can be used. However, it will now be shown that also an optimal integer aperture (OIA) estimator exists.

As with integer estimation, the optimality property would be to maximize the success rate, but in this case for a fixed failure rate. So, the optimization problem is to determine the aperture space which fulfills: 


$$
\max _{\Omega_{0} \subset S_{0}} P_{S} \quad \text { subject to: } \quad P_{f}=\beta
$$

where $\beta$ is a chosen fixed value for the failure rate. The solution of the optimization problem is given by, cf.Teunissen (2003c, 2004):

$$
\Omega_{0}=\left\{x \in S_{0} \mid \sum_{z \in \mathbb{Z}^{n}} f_{\hat{a}}(x+z) \leq \mu f_{\hat{a}}(x+a)\right\}
$$

The best choice for $S_{0}$ is the ILS pull-in region.

Using equations (14) and (18) the optimal datadriven approach follows as:

1. Apply ILS to obtain $\check{a}$

2. Evaluate the probability densities $f_{\breve{\epsilon}}(\hat{a}-\check{a})$ and $f_{\hat{a}}(\hat{a}-\check{a})$

3. Determine $\mu$ based on the user-defined $P_{f}=\beta$

4. Decision:

$$
\text { If } \frac{f_{\check{\epsilon}}(\hat{a}-\check{a})}{f_{\hat{a}}(\hat{a}-\check{a})} \begin{cases}\leq \mu & \text { use } \check{a} \\ >\mu & \text { use } \hat{a}\end{cases}
$$

Compare this result with the approach using the Ratio Test in section 3.4. In both approaches the test statistics are defined as a ratio. In the case of the Ratio Test, it only depends on $\|\hat{a}-\check{a}\|_{Q_{\hat{a}}}^{2}$ and $\| \hat{a}-$ $\check{a}_{2} \|_{Q_{\hat{a}}}^{2}$, whereas from equation (18) it follows that the optimal test statistic depends on all $\|\hat{a}-z\|_{Q_{\hat{a}}}^{2}, z \in$ $\mathbb{Z}^{n}$ if it is assumed that the float solution is normally distributed.

In Teunissen and Verhagen (2004) the performance of the Ratio Test and Optimal integer aperture estimator were compared. It followed that often the Ratio Test performs close to optimal, provided that the fixed failure rate approach is used. Furthermore, with integer aperture estimation a shorter time to first fix can be obtained as compared to the model-driven approach of section 2 and the traditional Ratio Test with fixed critical value $\delta$, while at the same time it is guaranteed that the failure rate is below a userdefined threshold.

\section{References}

Euler, H. J. and Schaffrin, B. (1991). On a measure for the discernibility between different ambiguity solutions in the statickinematic GPS-mode. IAG Symposia no. 107,
Kinematic Systems in Geodesy, Surveying, and Remote Sensing. Springer-Verlag, New York, pages 285-295.

Han, S. and Rizos, C. (1996). Integrated methods for instantaneous ambiguity resolution using new-generation GPS receivers. Proceedings of IEEE PLANS' 96, Atlanta GA, pages 254-261.

Hofmann-Wellenhoff, B. and Lichtenegger, H. (2001). Global Positioning System: Theory and Practice. Springer-Verlag, Berlin, 5 edition.

Leick, A. (2003). GPS Satellite Surveying. John Wiley and Sons, New York, 3rd edition.

Misra, P. and Enge, P. (2001). Global Positioning System: Signals, Measurements, and Performance. Ganga-Jamuna Press, Lincoln MA.

Strang, G. and Borre, K. (1997). Linear Algebra, Geodesy, and GPS. Wellesley-Cambridge Press, Wellesley MA.

Teunissen, P. J. G. (1993). Least squares estimation of the integer GPS ambiguities. Invited lecture, Section IV Theory and Methodology, IAG General Meeting, Beijing.

Teunissen, P. J. G. (1998). GPS carrier phase ambiguity fixing concepts. In: PJG Teunissen and Kleusberg A, GPS for Geodesy, Springer-Verlag, Berlin.

Teunissen, P. J. G. (1999a). An optimality property of the integer least-squares estimator. Journal of Geodesy, 73(11), 587-593.

Teunissen, P. J. G. (1999b). The probability distribution of the GPS baseline for a class of integer ambiguity estimators. Journal of Geodesy, 73, 275-284.

Teunissen, P. J. G. (2002). The parameter distributions of the integer GPS model. Journal of Geodesy, 76(1), 41-48.

Teunissen, P. J. G. (2003a). Integer aperture GNSS ambiguity resolution. Artificial Satellites, 38(3), 79-88.

Teunissen, P. J. G. (2003b). Theory of integer aperture estimation with application to GNSS. MGP report, Delft University of Technology.

Teunissen, P. J. G. (2003c). Towards a unified theory of GNSS ambiguity resolution. Journal of Global Positioning Systems, 2(1), 1-12.

Teunissen, P. J. G. (2004). Penalized GNSS ambiguity resolution. Journal of Geodesy, 78(4-5), 235-244.

Teunissen, P. J. G. and Verhagen, S. (2004). On the foundation of the popular ratio test for GNSS ambiguity resolution. Proceedings of ION GNSS-2004, Long Beach CA, pages 2529-2540.

Verhagen, S. (2004). Integer ambiguity validation: an open problem? GPS Solutions, 8(1), 36-43.

Verhagen, S. and Teunissen, P. J. G. (2004). PDF evaluation of the ambiguity residuals. In: F Sansò (Ed.), V. Hotine-Marussi Symposium on Mathematical Geodesy, International Association of Geodesy Symposia, Vol. 127, Springer-Verlag.

Wei, M. and Schwarz, K. P. (1995). Fast ambiguity resolution using an integer nonlinear programming method. Proceedings of ION GPS-1995, Palm Springs CA, pages 1101-1110. 